Authors Postprint of:

Southgate DFL, Hill AM, Alexander S, Wallace AL, Hansen UN, Bull AMJ. The Range of Axial Rotation of the Glenohumeral Joint. J Biomech 42; (2009), 1307-1312.

Publishers version can be found here:

http://www.sciencedirect.com/science/journal/00219290

\title{
The Range of Axial Rotation of the Glenohumeral Joint
}

\author{
Dominic FL Southgate ${ }^{1,2}$, Adam M Hill ${ }^{1,4}$, Susan Alexander ${ }^{3}$, Andrew L Wallace ${ }^{3}$, Ulrich N Hansen ${ }^{2}$, Anthony MJ Bull ${ }^{1}$ \\ ${ }^{1}$ Dept. of Bioengineering, Imperial College London, UK \\ ${ }^{2}$ Dept. of Mechanical Engineering, Imperial College London, UK \\ ${ }^{3}$ Shoulder Unit, Hospital of St John and St Elizabeth, London, UK \\ ${ }^{4}$ Faculty of Medicine, Imperial College London, UK
}

Corresponding Author:

\author{
Dr Anthony Bull \\ Department of Bioengineering \\ Imperial College London \\ Room 4.31 Royal School of Mines \\ South Kensington Campus \\ London. SW7 2AZ. \\ United Kingdom. \\ Telephone: +442075945186 \\ Fax: +44 2075846897 \\ E-mail: a.bull@imperial.ac.uk
}

\begin{abstract}
There is a paucity of data in the literature on the restraining effects of the glenohumeral $(\mathrm{GH})$ ligaments; cadaveric testing is one of the best methods for determining the function of these types of tissues. The aim of this work was to commission a custom-made six degree of freedom joint loading apparatus and to establish a protocol for laxity testing of cadaveric shoulder specimens. Nine cadaveric shoulder specimens were used in this study and each specimen had all muscle resected leaving the scapula, humerus (transected at mid-shaft) and GH capsule. Specimens were mounted on the testing apparatus with the joint in the neutral position and at $30^{\circ}, 60^{\circ}$ and $90^{\circ} \mathrm{GH}$ abduction in the coronal, scapula and $30^{\circ}$ forward flexion planes. For each orientation, $0-1 \mathrm{Nm}$ in $0.1 \mathrm{Nm}$ increments was applied in internal/external rotation and the angular displacement recorded. The toe-region of the moment-displacement curves ended at approximately $\pm 0.5 \mathrm{Nm}$. The highest rotational range of motion for the joint was $140^{\circ}$ for $\pm 1.0 \mathrm{Nm}$ at $30^{\circ} \mathrm{GH}$ abduction in the scapula plane. The range of motion shifted towards external rotation with increasing levels of abduction. The results provide the optimum loading regime to precondition shoulder specimens and minimize viscoelastic effects in the ligaments prior to laxity testing $\left(>0.5 \mathrm{Nm}\right.$ at $30^{\circ} \mathrm{GH}$ abduction in any of the three planes). Knowledge of the mechanical properties of the $\mathrm{GH}$ capsuloligamentous complex has implications for modelling of the shoulder as well surgical planning and intervention.
\end{abstract}

\section{Introduction}

The glenohumeral joint forms part of a complex series of articulations that comprise the shoulder joint. The shoulder joint permits movement through a wider range of motion than any other articulation. Stability of this joint is provided through a combination of active and passive stabilisers, but the specific role of each factor is still not fully understood
(Bigliani et al., 1996). Identifying the individual contribution of each of these structures is crucial to defining the limits of normal glenohumeral movement. Movement beyond these limits can occur following a structural injury to the shoulder, or as a result of abnormal neuromuscular activation patterns or due to an inherent deficiency within the capsulolabral tissues. Repeated episodes of excessive translation may lead to clinical instability that causes progressive structural damage to the joint (Bahk et al., 2007).

In-vitro mechanical testing of cadaveric shoulder specimens is a useful method that can be used for determining laxity and the mechanical properties of individual structures in a capsuloligamentous complex. Limitations that cannot be avoided with cadaveric experiments include the use of elderly cadavers to represent the general population and possible effects on soft tissue from freezing and fluid loss (Chimich et al., 1992; Lee et al., 1999; Hansen et al., 2006). There are, however, limitations that can be avoided. One significant limitation of previous testing set-ups has been coupled forces and translations. This can be solved by designing test apparatus that is able to translate and rotate through six independent degrees of freedom (dof). The shoulder specimen also has to be constrained in such a way as to enable testing in clinically relevant positions but not placing any restrictions that would prohibit physiological motion of the joint. It should have the capacity to record any load applied to the specimen and measure any resulting motion. This needs to be performed along clearly defined axes with a robust co-ordinate system for each bone so that the position of the joint can be related back to the anatomy in-vivo.

There have been a number of studies investigating the role of the passive stabilisers (Soslowsky et al., 1997; Moskal et al., 1999; Motzkin et al., 1998; Kuhn et al., 2000; Huffman et al., 2006) and some have focused on rotational laxity of the 
glenohumeral joint in isolated positions (Harryman et al., 1992; Kuhn et al., 2005; Jansen et al., 2006; Karduna et al., 1996; Fitzpatrick et al., 2005; Gerber et al., 2003; Alberta et al., 2006). However, there is little information on how the capsuloligamentous complex affects the axial rotation of the humerus through different planes and levels of abduction.

Soft tissues such as ligaments have viscoelastic properties (Woo, 1982; Schatzmann et al., 1998) which must be considered when conducting mechanical tests on cadaveric specimens to ensure a consistent methodology. A common way to achieve this is by preconditioning: cyclically loading the specimen prior to recording the data so that the "stretch" in the capsule is removed. However, the optimum position in which to conduct this pre-conditioning is rarely considered and the effect on each part of the capsule is not known. This study set out to establish the joint position (abduction and plane of abduction) where the range of humeral axial rotation is greatest and to describe the relative contribution of internal and external rotation to this range. It is hypothesised that at this point the loading on the different parts of the capsule is approximately equal. This position would then correspond to the optimum position in which to conduct pre-conditioning. This hypothesis is not tested in this work and is based on a series of assumptions. These are that the capsular ligaments are of a similar length (Bigliani et al., 1992), and that they insert in an ordered way around the glenoid that corresponds to the order in which they insert on the humeral anatomical neck. This means that it is assumed that the ligament paths do not cross each other within the capsule. Therefore, an axial rotation of the humerus would serve to either tighten or slacken all the capsular structures. The abduction position of the humerus and the plane of abduction can then be optimised to find the greatest range of rotation.

The purpose of this study was to study humeral axial internal and external rotation in different glenohumeral joint positions. In addition, this will establish the optimum position of the joint for pre-conditioning so that the effect would be consistent for all of the capsular structures in later studies.

\section{Materials and Methods}

A cadaveric-experimental approach was taken that included mounting humerus and scapula in a consistent manner in a 6 dof testing rig followed by rotational laxity testing.

\section{Specimens}

Ethical approval for this study was obtained from the Riverside Research Ethics Committee (Charing Cross Hospital, London, UK). Nine unpaired shoulders (5 male, 5 right, mean age 61, range 47-70) were used in this study and stored at $-20^{\circ} \mathrm{C}$ until required and then thawed for at least 12 hours before preparation and testing.

\section{Definition of anatomical axes}

The plane of the scapula was referenced using the inferior angle, supraglenoid tubercle and most medial protuberance of the scapula spine. This scapula plane was defined as vertical in its anatomical position and $30^{\circ}$ in front of the coronal plane. To define the rotation within this plane, the medial border was assumed to be vertical when the arm was in the neutral position (parallel to the humerus). A constant ratio of 2:3 was then used between glenohumeral $(\mathrm{GH})$ abduction and thoracohumeral (TH) abduction to approximate the kinematics of the shoulder rhythm (van der Helm, 1994; Barnett et al., 1999).
The coordinate system applied to this work is defined through a 3-cylinder open-chain mechanism (Grood and Suntay, 1983); the scapula frame is composed of the Z-axis embedded in the lateral border of the scapula directed towards the glenoid fossa, the Y-axis which is the righthanded cross product of the $\mathrm{Z}$-axis and a vector perpendicular to the face of the glenoid, and the $X$-axis which is the cross-product of $\mathrm{Y}$ and $\mathrm{Z}$ axes. As such, abduction is measured about the $\mathrm{X}$-axis. This coordinate frame is determined by the customised scapula mounting jig. The humeral long axis is defined by its intramedullary canal; the absence of epicondyles in the specimens meant that a reference point for rotation could not be taken. Zero rotation was therefore not defined prior to the loading regime.

\section{Specimen preparation}

Each specimen initially featured only scapula and humerus with intact rotator cuff musculature and no additional covering soft tissues. The humerus was transected mid-shaft. All musculature was removed and care was taken not to disrupt the interface between the rotator cuff and the capsuloligamentous complex. A casting box jig was constructed from aluminium for potting the scapula to enable accurate and repeatable setting of each specimen in bone cement (Figure 1) with reference to the anatomical axes described above, where the inferior angle, most medial protuberance of the scapular border and supraglenoid tubercle were mounted in a central plane in the casting box and the medial border parallel to the superior-inferior axis of the casting box. This device features stainless steel location pins and a sliding stylus to set the medial border alignment and the rotation of the scapula within its plane consistently between shoulders. Once correctly orientated, the scapula is held in place by a set of screws whilst the jig is reassembled to form an open box that is then filled with bone cement.

The humerus was reamed to accept an $8 \mathrm{~mm}$ intramedullary rod fixed with bone cement. Once set, the specimen was transferred to the laxity testing apparatus for the experiments, and at all times kept well hydrated using physiological saline.

\section{Laxity testing apparatus}

A custom-made rig allows independent motion for six dof to be provided; three rotations and three translations (Figure 2). The three rotational degrees allow the joint to be manipulated into any physiological position that may be encountered in a clinical setting, through abduction, changing the plane of abduction (flexion) and axial rotation. The three translational degrees then allow the scapula and humerus to be moved relative to each other in orthogonal axes representing mediolateral $(\mathrm{M} / \mathrm{L})$, anteroposterior $(\mathrm{A} / \mathrm{P})$ and superoinferior (S/I) directions. Two of the three rotations, abduction and the plane of abduction, are constrained but the third, axial rotation, has the option to be unconstrained, or controlled. This means that a moment can be applied in internal or external rotation and the angular displacement measured. 

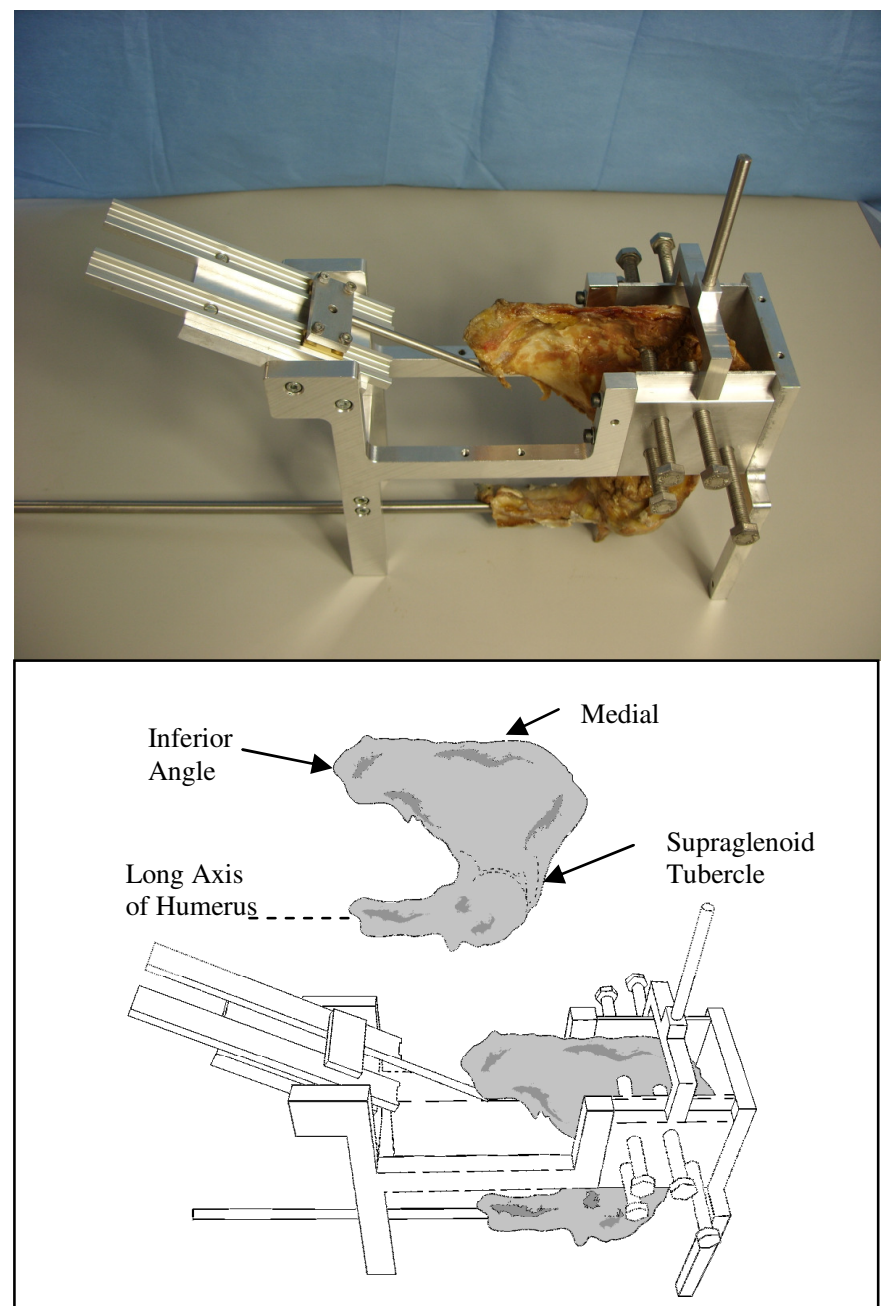

Figure 1 - The potting jig and specimen with intramedullary rod fitted. The labelled anatomical landmarks are used to reference the scapula and fix its orientation before trimming and setting in bone cement.

The three slide-beds for the scapula attachment allow independent translation of the joint along each axis. These translations are also active so that a force can be applied and linear displacement measured; these can be constrained if required. Each slide-bed is constrained to a single axis of motion by linear bearings running on parallel shafts and the load is applied through a pulley system and suspended weights. A moment is applied to the humerus in axial rotation via loads suspended from a pulley on the intramedullary rod.

Abduction of the joint is specified by adjusting the angle of the scapula mounting box to pre-marked positions on the frame. The plane of abduction is altered by moving the humeral mounting along a semi-circular track and measured using a handheld protractor. The displacement of each active dof is tracked in real-time; the linear translations of each slide-bed are measured using linear variable differential transformers (LVDTs - Solartron Metrology, Bognor Regis, $\mathrm{UK}, \pm 0.1 \mathrm{~mm})$. The axial rotation of the humerus is measured using a rotary potentiometer attached to the end of the intramedullary rod and recorded to the nearest whole degree. The data from each transducer are then recorded through a connector box (BNC-2120, National Instruments, Austin, TX, USA), data acquisition card (NI6023E, National Instruments) in a PC and processed using data capture software in real-time (LabVIEW, National Instruments, Austin, TX, USA).

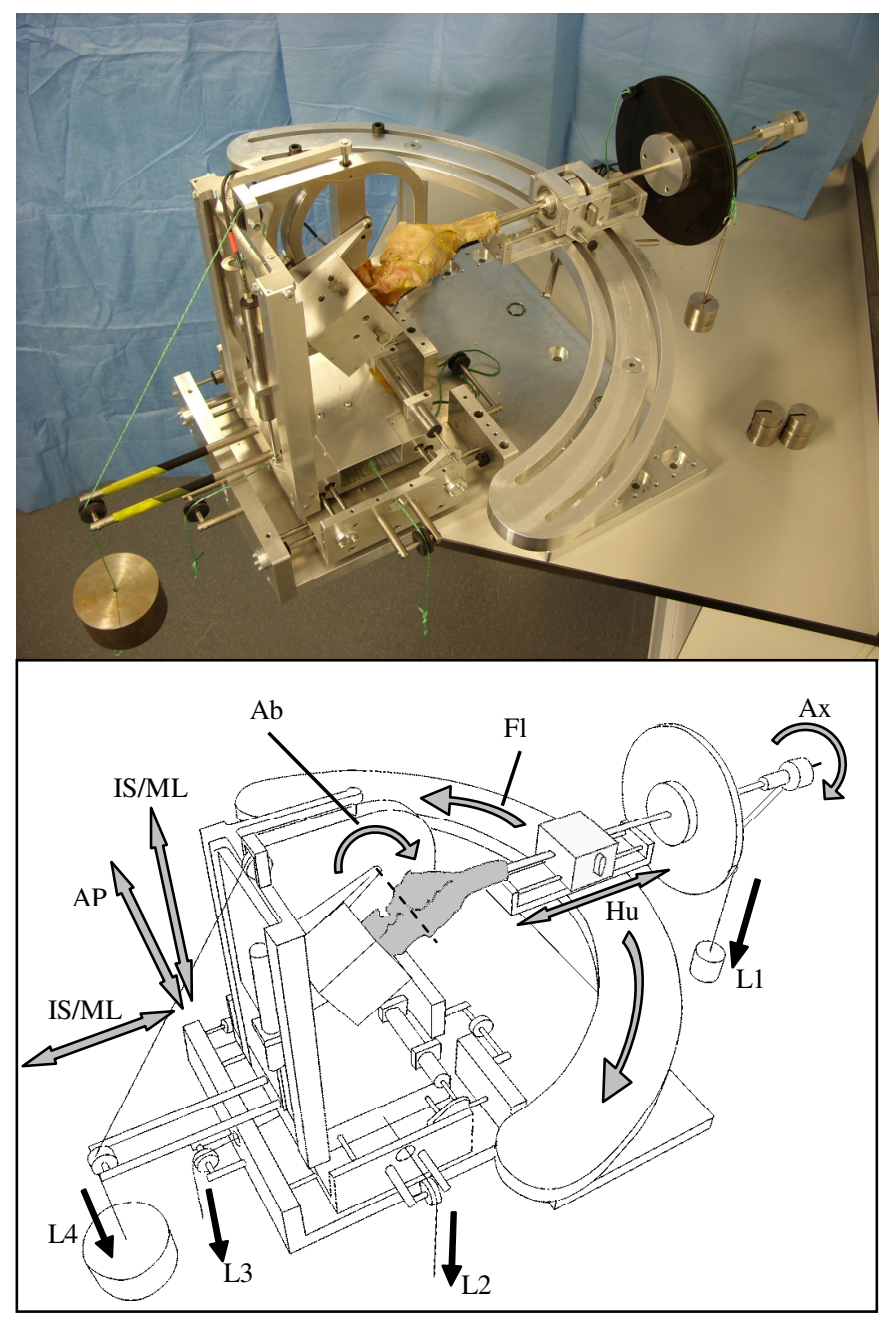

Figure 2 - The laxity testing apparatus showing the six degrees of freedom, three translations: IS - Inferosuperior, ML Mediolateral, AP - Anteroposterior and three rotations: Ab Abduction, Fl - Flexion (or plane of abduction), Ax - Axial rotation. An additional degree of freedom was included to allow humeral axial translation (Hu). In this study, the humerus was rotated both internally and externally by applying loads to the pulley on the intramedullary rod, L1. The system also has the capability to translate the scapula in AP/IS/ML directions by applying loads through pulleys L2, L3 and L4.

\section{Loading protocol}

In this study no translations were applied or recorded. No compressive load was applied to the joint, because it would have caused an additional moment about that axis varying with the rotation of the humerus.

Pilot tests confirmed that a $1 \mathrm{Nm}$ internal/external rotation moment would not cause excessive damage to the joint capsule, but would be in the stiff region of the curve of rotational laxity. This was defined as a change of less than $3^{\circ}$ in rotation over a $0.1 \mathrm{Nm}$ increase in moment. Each specimen was positioned on the laxity testing apparatus in the neutral position and loaded with a moment of $1 \mathrm{Nm}$ in internal and external rotation for 10 cycles to ensure free movement of the joint up to this load. The joint was then manipulated to ensure that it was resting in a reduced position without any load and the rotation measurement was zeroed. The joint was then loaded in internal and external rotation with a moment from $0-1 \mathrm{Nm}$ in $0.1 \mathrm{Nm}$ increments and the angular displacement at each step was recorded. This loading regime was then repeated with the joint at $30^{\circ}, 60^{\circ}$ and $90^{\circ} \mathrm{GH}$ abduction, in the scapula plane, coronal plane and $30^{\circ}$ forward of the scapula plane. On completion of these tests, the loading regime was repeated for the neutral position. 
The rotational loads were applied manually but always by the same investigator and at an approximate loading rate of $0.05 \mathrm{Nm} / \mathrm{s}$. Each load was left for 10 seconds before the displacement reading was taken and the next load was applied, to ensure a consistent method. The specimens were observed throughout the duration of the experiments and any visual tightening of the capsuloligamentous complex was recorded.

\section{Data analysis}

The data points for each specimen in the neutral position were averaged to calculate the mean offset of the curve from the zeroed position during the experiment. This offset was then subtracted from all the curves for that specimen to normalise the data and enable a comparison between all 9 datasets.

The mean angular displacement and standard deviation using all the specimens was calculated for each load and joint position. Paired-samples t-tests were used to examine any differences between the initial and post-test repeat in the neutral position for $1 \mathrm{Nm}$ load in both internal and external rotation. A two-way ANOVA was conducted to assess the effect of joint abduction angle and plane on joint positions under $\pm 1 \mathrm{Nm}$ load. The significance level was set at $\mathrm{p}=0.05$.

\section{Results}

Data for one specimen were lost due to technical difficulties, leaving eight specimens for analysis. The average loading curve for the joint in neutral position showed a slack region in both internal and external rotation followed by a stiffer section. This shape was seen to a greater or lesser extent for each testing position. At the highest level of $\mathrm{GH}$ abduction $\left(90^{\circ}\right)$ this slack region was reduced and the curve took a more linear form passing from internal through external rotation. With the joint in $90^{\circ} \mathrm{GH}$ abduction the whole capsuloligamentous complex, and especially the inferior portion of the capsule, visibly tightened due to the limiting length of the structures. The onset of the stiffer region, defined as a region where the axial rotation increased by less than $3^{\circ}$ per $0.1 \mathrm{Nm}$ increase in axial moment, occurred at approximately $0.5 \mathrm{Nm}$ in each position (Figure 3 ).

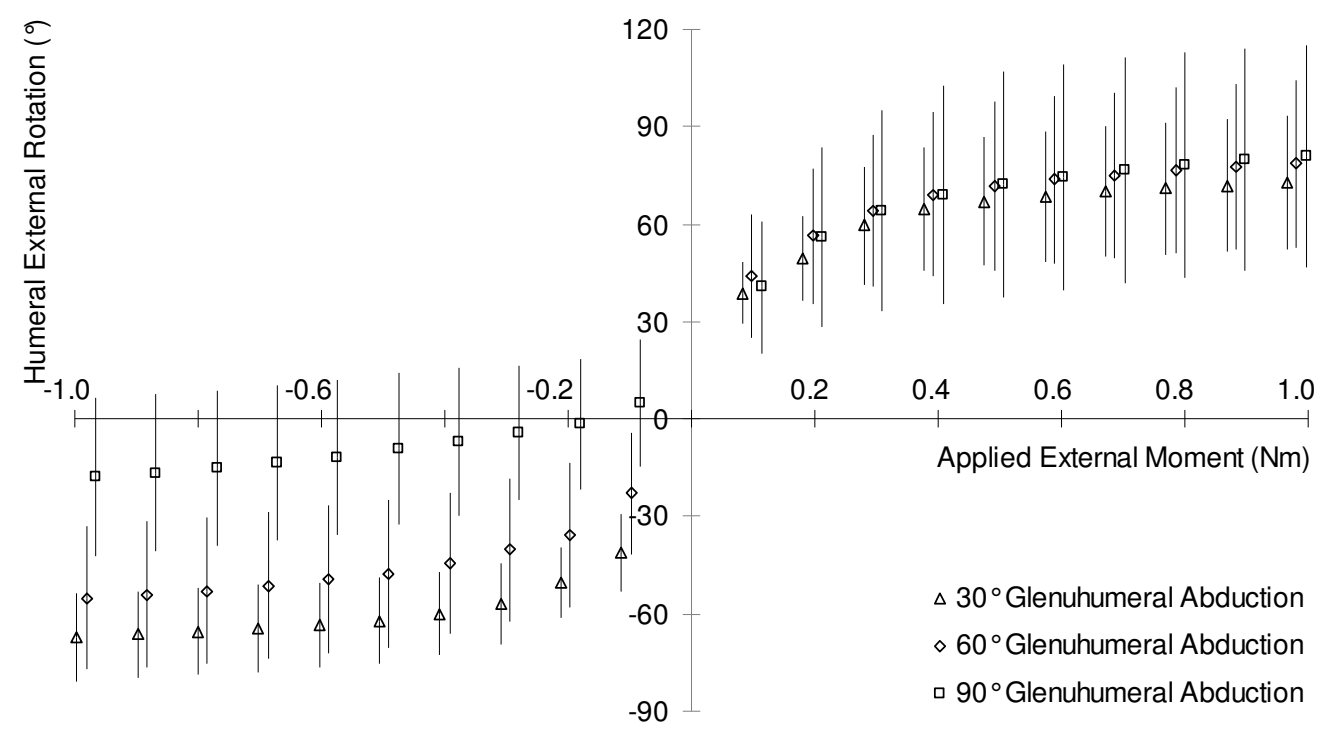

Figure 3 - Average rotational range of motion in the scapula plane at different levels of abduction (n=8).

Rotation results are presented in Table 1. The level of abduction produced a significant difference in external rotation $(p=0.0017)$ and internal rotation $(p<0.0001)$. The plane of abduction produced a significant difference in external rotation $(\mathrm{p}=0.0436)$ but not in internal rotation $(\mathrm{p}=0.0513)$. Therefore, as abduction increased there was a decrease in the range of rotation and a shift towards external rotation. There was no significant difference in angular displacement between the initial and post-test repeat in the neutral position i.e. the specimens were not significantly stretched during the course of the loading protocol $(\mathrm{p}=0.16$ for external rotation, $\mathrm{p}=0.67$ for internal rotation).

Angle of GH Abduction

\begin{tabular}{cc|ccccc} 
Plane & & $0^{\circ}$ (Initial) & $0^{\circ}$ (Repeat) & $30^{\circ}$ & $60^{\circ}$ & $90^{\circ}$ \\
\hline $30^{\circ}$ Forward & ER & - & - & $74(19)$ & $81(18)$ & $89(27)$ \\
& IR & - & - & $-60(15)$ & $-44(18)$ & $-9(21)$ \\
& Total & - & - & $134(29)$ & $125(32)$ & $99(43)$ \\
\hline \multirow{2}{*}{ Scapula } & ER & $60(12)$ & $63(11)$ & $73(20)$ & $79(26)$ & $75(30)$ \\
& IR & $-58(11)$ & $-57(10)$ & $-67(13)$ & $-55(22)$ & $-18(24)$ \\
& Total & $118(22)$ & $119(21)$ & $140(31)$ & $134(46)$ & $93(50)$ \\
\hline \multirow{2}{*}{ Coronal } & ER & - & - & $70(25)$ & $59(30)$ & $71(33)$ \\
& IR & - & - & $-68(12)$ & $-61(18)$ & $-30(24)$ \\
& Total & - & - & $138(33)$ & $119(44)$ & $101(51)$
\end{tabular}

Table 1 - Comparison of the average rotational range of motion in degrees $( \pm \mathrm{SD})$ in different joint positions with $\pm 1 \mathrm{Nm}$ applied moment. 


\section{Discussion}

In this preliminary study of rotational laxity of the glenohumeral joint a consistent kinematic pattern was observed for all of the specimens tested. No significant changes occurred during the course of the loading protocol and so the method was valid. The type of response to be expected from loading cadaveric shoulder specimens in humeral axial rotation has been established.

The loading curves in all joint orientations demonstrated a slack region, followed by a near-linear section in both internal and external rotation. This is not a toe-region as in a material property but an area where the moment is simply rotating the joint until the capsule becomes taught. The moment required to reach the stiffer region of the loading curve was approximately $0.5 \mathrm{Nm}$ in both internal and external rotation. This has been demonstrated previously by Kuhn et al. (2005), although they found that this stiffer region began at $1.1 \mathrm{Nm}$ of applied moment. However, this was for a specific loading protocol that also featured muscle loads applied to the rotator cuff tendons, which could cause additional restraining effects on the joint varying with humeral rotation.

The anatomy of the proximal humerus is such that the centre of rotation for the humeral head is offset from the long axis of the bone. A compressive load on the humeral head applied perpendicular to the glenoid therefore causes an additional moment about the long axis when the glenoid surface is not perpendicular to the humeral long axis (Figure 4). This occurs for any position other than when the line joining the centre of rotation of the humeral head and the long axis is perpendicular to the face of the glenoid. If the compressive force were applied as an axial load through the humerus, stability would be conferred with the joint in the region of $90^{\circ} \mathrm{GH}$ abduction, but at lower levels of abduction would act to translate the humeral head superiorly out of the glenoid. For these reasons, a compressive joint load should not be used when assessing passive rotation of the glenohumeral joint.

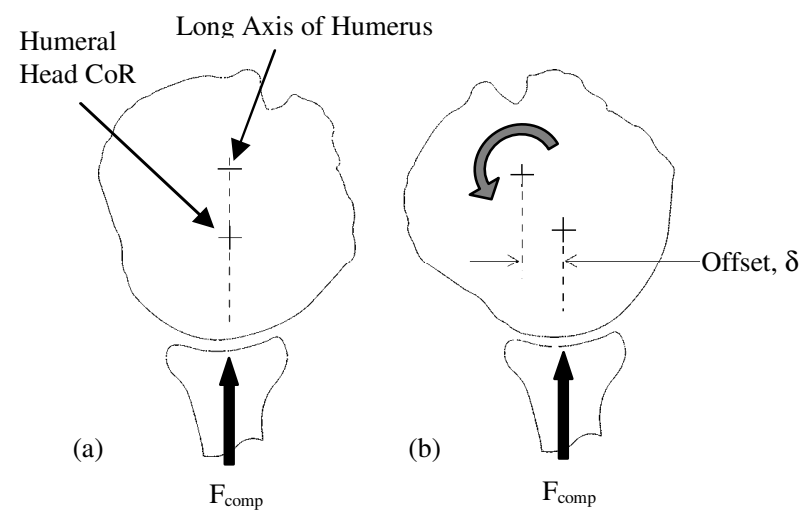

Figure 4a,b - Superior view of the glenohumeral joint. In (a) the compressive joint load is in line with the centre of rotation (CoR) of the humeral head and the long axis of the humerus. In (b) the humerus is rotated internally and the humeral head CoR becomes offset from the long axis so that the compressive joint load causes an additional moment about the long axis.

The greatest range of motion was $140^{\circ}$ and found when the joint was at $30^{\circ} \mathrm{GH}$ abduction in the scapula plane. This is similar to Jansen et al. (2006) who also found that the maximum range of humeral rotation occurred at $30^{\circ} \mathrm{GH}$ abduction in the scapula plane. Their range of motion was less $\left(101^{\circ}\right)$ despite a higher applied moment of $1.8 \mathrm{Nm}$. Their maximal internal rotation was seen at low levels of $\mathrm{GH}$ abduction $\left(15^{\circ}\right)$ and at maximal abduction the rotational range was greatly restricted. These are in correspondence with our findings. The observed tightening of the inferior portion of the capsule at $90^{\circ} \mathrm{GH}$ abduction in our experiments provides a possible explanation for the narrowing of the slack region and the more linear path between internal and external rotation in this joint position.

The range of motion found in this study is in general agreement with data published by Karduna et al. (1996), who found a rotational range of approximately $140^{\circ}$ for the joint at $60^{\circ} \mathrm{GH}$ abduction in the scapula plane, although the applied moment was unspecified. Alberta et al. (2006) found a higher rotational range $\left(170^{\circ}\right)$ with a higher applied moment $(2.2 \mathrm{Nm})$. In the neutral position Harryman et al. (1992) found a similar mean rotational range of motion of $128.5^{\circ}$ under $1.5 \mathrm{Nm}$ of moment. Fitzpatrick et al. (2005) looked at the rotational range of motion in two cadaveric models of a thrower's shoulder with the GH joint abducted to $60^{\circ}$. They found a rotational range of $136^{\circ}$ and $153^{\circ}$ using $2.0 \mathrm{Nm}$ moment but also with a $22 \mathrm{~N}$ compressive joint load.

The effect of the passive stabilisers to shift the range of motion towards external rotation with increasing abduction of the humerus is an interesting finding that has received little attention in the literature, although it is known to the clinician who is able to achieve this with the patient under anaesthesia by passive manipulation. The kinematics associated with this motion would be expected in-vivo, as the humerus must externally rotate to maintain contact between the articular surface of the humeral head and the glenoid at the extremes of arm abduction and also to avoid contact between the greater tubercle and the acromion.

This effect has been repeated, although not discussed, by Gerber et al. (2003). However, the results of their experiments differ slightly from the current study as they found a much larger increase in external rotation with increasing glenohumeral abduction. They investigated $0^{\circ}$, $30^{\circ}$ and $60^{\circ} \mathrm{GH}$ abduction in the scapula plane with a 0.5 $\mathrm{Nm}$ applied moment and found that the mean internal rotation decreased from $45^{\circ}$ to $39^{\circ}$ to $31^{\circ}$ but external rotation increased from $53^{\circ}$ to $104^{\circ}$ to $133^{\circ}$ over the three positions.

This testing apparatus can be used for characterising the envelope of laxity under many different loading conditions, as well as simulating clinical tests and assessing the biomechanics of glenohumeral joint pathology and repair. Examples of future work could include investigating the effects of labral pathology and repair on the passive stability of the joint as well as more basic scientific studies on the envelope of laxity of the joint in both translation (anteriorposterior and superior-inferior) and rotation.

An important step before commencing these further experiments was to establish a routine for pre-conditioning of the specimens; to minimise the impact of the viscoelastic properties of the capsule whilst ensuring that it is equal for all passive structures of the joint. Cyclically loading the joint in internal/external rotation should pre-condition the entire capsuloligamentous complex at the same time due to the spiral wrapping around the humeral head, assuming that the capsular structures are of similar lengths. The most even loading of the capsule will occur when the joint exhibits the greatest range of motion, as no single band is recruited 
before the others preventing further rotation of the joint. A joint position of $30^{\circ}$ abduction in any of the three planes tested will therefore be most suitable for preconditioning specimens in future laxity experiments.

This study has described the use of a laxity testing apparatus for human cadaveric shoulder joints. The optimal abduction angle to precondition the capsule in humeral rotation has been defined and it has been demonstrated that the envelope of humeral axial rotation shifts externally as the humerus abducts.

\section{Acknowledgements}

This study was funded by the Furlong Research Charitable Foundation, the Arthritis Research Campaign and the EPSRC.

The sponsors had no involvement with the design of the study, or the collection, analysis and interpretation of the data.

\section{Reference List}

Alberta, F. G., Elattrache, N. S., Mihata, T., McGarry, M. H., Tibone, J. E., Lee, T. Q., (2006). Arthroscopic anteroinferior suture plication resulting in decreased glenohumeral translation and external rotation. Study of a cadaver model. The Journal Of Bone And Joint Surgery.American Volume 88, 179-187.

Bahk, M., Keyurapan, E., Tasaki, A., Sauers, E. L., McFarland, E. G., (2007). Laxity Testing of the Shoulder: A Review. American Journal of Sports Medicine 35, 131-144.

Barnett, N. D., Duncan, R. D. D., Johnson, G. R., (1999). The measurement of three dimensional scapulohumeral kinematics - a study of reliability. Clinical Biomechanics 14, 287-290.

Bigliani, L. U., Kelkar, R., Flatow, E. L., Pollock, R. G., Mow, V. C., (1996). Glenohumeral stability. Biomechanical properties of passive and active stabilizers. Clinical Orthopaedics And Related Research 13-30.

Bigliani, L. U., Pollock, R. G., Soslowsky, L. J., Pawluk, R. J., Mow, V. C., (1992). Tensile properties of the inferior glenohumeral ligament. Journal of Orthopaedic Research; 10, 187-197.

Chimich, D., Shrive, N., Frank, C., Marchuk, L., Bray, R., (1992). Water content alters viscoelastic behaviour of the normal adolescent rabbit medial collateral ligament. Journal of Biomechanics 25, 831-833.

Fitzpatrick, M. J., Tibone, J. E., Grossman, M., McGarry, M. H., Lee, T. Q., (2005). Development of cadaveric models of a thrower's shoulder. Journal of Shoulder and Elbow Surgery 14, S49-S57.

Gerber, C., Werner, C. M. L., Macy, J. C., Jacob, H. A. C., Nyffeler, R. W., (2003). Effect of Selective Capsulorrhaphy on the Passive Range of Motion of the Glenohumeral Joint. Journal of Bone and Joint Surgery 85, 48-55.

Grood, E. S., Suntay, W. J., (1983). A joint coordinate system for the clinical description of three-dimensional motions: application to the knee. Journal Of Biomechanical Engineering 105, 136-144.

Hansen, U., Masouros, S., Amis, A. A., (2006). Material properties of biological tissues related to joint surgery. Current Orthopaedics 20, 16-22.

Harryman, D. T., Sidles, J. A., Harris, S. L., Matsen, F. A., (1992). The role of the rotator interval capsule in passive motion and stability of the shoulder. Journal of Bone and Joint Surgery 74-A, 53-66.

Huffman, G. R., Tibone, J. E., McGarry, M. H., Phipps, B. M., Lee, Y. S., Lee, T. Q., (2006). Path of Glenohumeral Articulation Throughout the Rotational Range of Motion in a Thrower's Shoulder Model. American Journal of Sports Medicine 34, 1662-1669.

Jansen, J. H. W., de Gast, A., Snijders, C. J., (2006). Glenohumeral elevation-dependent influence of anterior glenohumeral capsular lesions on passive axial humeral rotation. Journal of Biomechanics 39, 1702-1707.

Karduna, A. R., Williams, G. R., Iannotti, J. P., Williams, J. L., (1996). Kinematics of the glenohumeral joint: influences of muscle forces, ligamentous constraints, and articular geometry. Journal of Orthopaedic Research 14, 986-993.

Kuhn, J. E., Bey, M. J., Huston, L. J., Blasier, R. B., Soslowsky, L. J., (2000). Ligamentous Restraints to External Rotation of the Humerus in the Late-Cocking Phase of Throwing: A Cadaveric Biomechanical Investigation. American Journal of Sports Medicine 28, 200-205.

Kuhn, J. E., Huston, L. J., Soslowsky, L. J., Shyr, Y., Blasier, R. B., (2005). External rotation of the glenohumeral joint: Ligament restraints and muscle effects in the neutral and abducted positions. Journal of Shoulder and Elbow Surgery 14, S39-S48.

Lee, T. Q., Dettling, J., Sandusky, M. D., McMahon, P. J., (1999). Age related biomechanical properties of the glenoidanterior band of the inferior glenohumeral ligament-humerus complex. Clinical Biomechanics 14, 471-476.

Moskal, M. J., Harryman II, D. T., Romeo, A. A., Rhee, Y. G., Sidles, J. A., (1999). Glenohumeral Motion After Complete Capsular Release. Arthroscopy: The Journal of Arthroscopic \& Related Surgery 15, 408-416.

Motzkin, N. E., Itoi, E., Morrey, B. F., An, K. N., (1998). Contribution of capsuloligamentous structures to passive static inferior glenohumeral stability. Clinical Biomechanics 13, 54-61.

Schatzmann, L., Brunner, P., Staubli, H. U., (1998). Effect of cyclic preconditioning on the tensile properties of human quadriceps tendons and patellar ligaments. Knee Surgery, Sports Traumatology, Arthroscopy 6, S56-S61.

Soslowsky, L. J., Malicky, D. M., Blasier, R. B., (1997). Active and passive factors in inferior glenohumeral stabilization: A biomechanical model. Journal of Shoulder and Elbow Surgery 6, 371-379.

van der Helm, F. C. T., (1994). Analysis of the kinematic and dynamic behavior of the shoulder mechanism. Journal of Biomechanics 27, 527-550.

Woo, S. L., (1982). Mechanical Properties of Tendons and Ligaments.1. Quasi-Static and Non-Linear Viscoelastic Properties. Biorheology 19, 385-396. 